BRAIN IMAGING

\section{Synaptic density directly visualized in human brains}

Synaptic density has been directly visualized in living human brains for the first time in new work that demonstrates the potential of a novel PET tracer. The approach enabled detection of synaptic loss in patients with epilepsy, and could provide new insights into a variety of neurological diseases.

Synaptic pathology is associated with many neurological disorders,

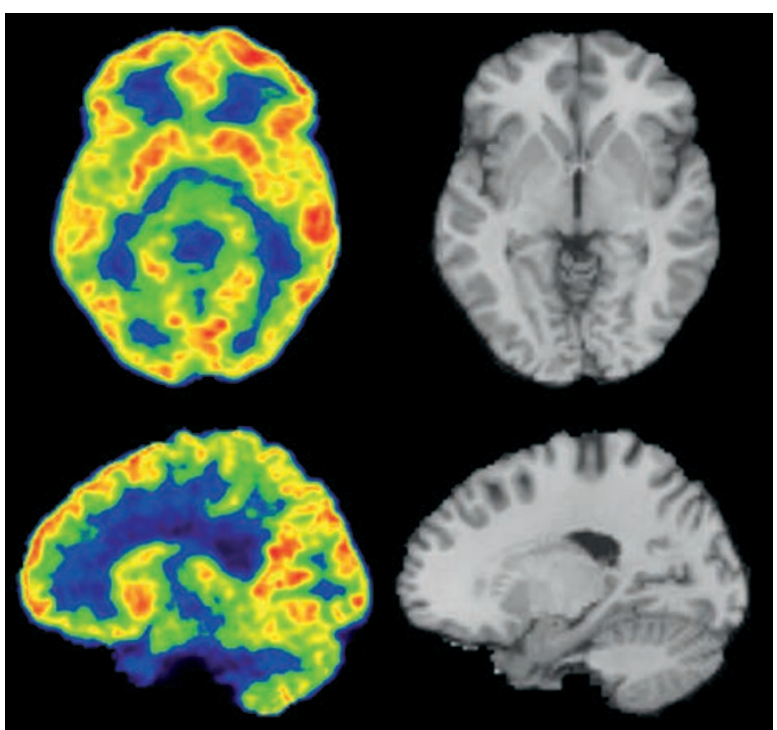

${ }^{11} \mathrm{C}-\mathrm{UCB}-J-P E T$ summation images at 40-60 min after injection in a single healthy subject (left) and corresponding MRI (right). Image courtesy of Sjoerd J. Finnema. but quantification of synapses in human brains currently requires autopsy specimens or surgical resection. PET imaging of synapses could facilitate our understanding of synaptic changes over a lifetime and in disease, but a PET tracer that targets a ubiquitous synaptic protein has been lacking.

Richard Carson, Sjoerd Finnema and colleagues previously developed ${ }^{11} \mathrm{C}-\mathrm{UCB}-\mathrm{J}$, a radioligand targeted to synaptic vesicle glycoprotein $2 \mathrm{~A}$ (SV2A). " ${ }^{11} \mathrm{C}-\mathrm{UCB}-\mathrm{J}$ could be a general-purpose synaptic density marker because SV2A is ubiquitously present in presynaptic terminals," says Carson.

To evaluate the potential of ${ }^{11} \mathrm{C}-\mathrm{UCB}-\mathrm{J}$ as a synaptic PET tracer, Finnema and colleagues first validated it as a marker of synaptic density in baboons before testing it for the first time in humans. They then used ${ }^{11} \mathrm{C}$-UCB-J-PET to image the brains of ten healthy controls and three patients with epilepsy. The researchers demonstrated that ${ }^{11} \mathrm{C}$-UCB-J was effective for PET imaging of synapses in humans, and enabled the detection of synaptic loss in patients with temporal lobe epilepsy.
"Our study was the first human brain imaging study with a tracer for SV2A," says Finnema. "Synaptic loss in patients with epilepsy has previously been studied with PET using ${ }^{18} \mathrm{~F}$-FDG to measure glucose consumption, an index related to synaptic density. The ${ }^{11} \mathrm{C}-\mathrm{UCB}-\mathrm{J}$ signal is expected to be a more specific to synaptic loss than ${ }^{18} \mathrm{~F}-\mathrm{FDG}$, although a full comparative study needs to be performed."

Carson anticipates that larger cohort studies over the next 6-12 months will further validate ${ }^{11} \mathrm{C}-\mathrm{UCB}-\mathrm{J}$ as a synaptic PET tracer, and the next challenge will be to assess the value of ${ }^{11} \mathrm{C}-\mathrm{UCB}-\mathrm{J}-\mathrm{PET}$ in other brain disorders, such as Parkinson disease, Alzheimer disease (AD), depression and schizophrenia.

"Of particular interest is the evaluation of patients with AD," says Carson. "Synaptic loss is thought to be closely related to cognitive dysfunction, and longitudinal evaluation of amyloid- $\beta$, tau tangles and synaptic loss would be of great value for staging of disease progression. There are already established PET tracers for amyloid- $\beta$ and tau, and we now add ${ }^{11} \mathrm{C}$-UCB-J for synaptic density imaging."

Alan W. J. Morris

ORIGINAL ARTICLE Finnema, S. J. et al. Imaging synaptic density in the living human brain. Science Translational Medicine http://dx.doi.org/10.1126/ scitranslmed.aaf6667 (2016) 